\title{
Micromachined Fluidic Couplers
}

\author{
Ellis Meng, Shuyun Wu, and Yu-Chong Tai \\ California Institute of Technology, Caltech Micromachining Laboratory, 136-93, \\ Pasadena, CA 91126
}

\begin{abstract}
A family of self-aligning micromachined fluidic couplers are presented here. Bulk micromachined and DRIE processed silicon structures have been investigated as a means of providing external connections via fused silica capillaries for MEMS fluidic devices. These couplings can have arbitrary geometry, ensuring compatibility with most fluidic ports. In addition, performance exceeds typical requirements with an operating range of at least 0 1200 psi.
\end{abstract}

\section{Keywords: microfluidics, fluidic couplers, fluidic interconnects}

\section{Introduction}

MEMS fluidic devices have long been plagued by the lack of an efficient and convenient means of connection to the outside world or even to other MEMS devices. Many micromachined fluidic devices incorporate connections formed by manually gluing tubes to I/O ports. Disadvantages of this method include low yield, complex assembly, misalignment, frequent failure, and difficulty to salvage used components. In addition, this method requires a significant footprint and is not universally compatible. Several different approaches have been developed [1-3], however the same critical issues exist. Micromachined rubber o-ring couplers [4] are reusable but feasible only for lower pressures. To address the above issues, device-independent prefabricated fluidic couplers have been conceived. A comparison of traditional manual coupling techniques with our new approach is demonstrated on thermopneumatic valves from [5] in Figure 1.

Micromachined fluidic couplers consist of a silicon fitting with a sleeve into which a capillary is press-fitted. Fittings are designed to include alignment structures and if necessary, stepped sleeves that serve as a tubing insertion depth guide. Couplers are joined to pre-existing fluidic ports via these fittings. A variety of joining methods including adhesive, solder, and eutectic bonding can be implemented at the coupler-to- $\mathrm{I} / \mathrm{O}$ port interface. Alternatively, couplers can also be molded with a thermoplastic material and subsequently reflowed to form a connection. Modified couplers with fitting structures at both ends of the capillary can also be used to concatenate microfluidic devices.

\section{Design}

Fluidic ports are typically created by anisotropic etching, drilling, or DRIE (deep reactive ion etching). The geometries associated with these fabrication methods dictate coupler design. Various coupler designs stem from two common geometries (Figure 2). The first incorporates the truncated pyramidal geometry resulting from anisotropic etching. While it is possible to glue tubing directly to pyramidal pits, leakage paths due to mismatch (noninterlocking halves) result in clogging when using less viscous adhesives. In addition, each of these structures can be designed with a shoulder to constrain the usable height of the coupler. The second scheme can be used with fluidic ports of circular cross-section that are formed by drilling or DRIE and are currently being fabricated. Furthermore, by using DRIE, 


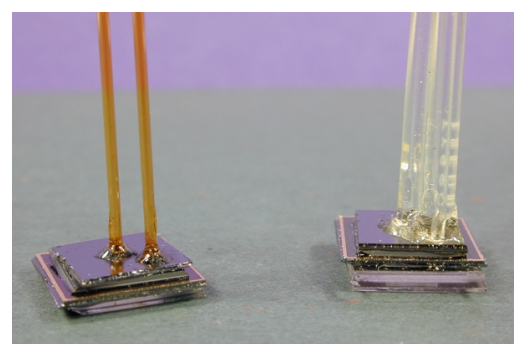

Figure 1

Comparison of Techniques

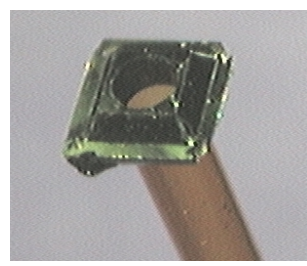

(a)

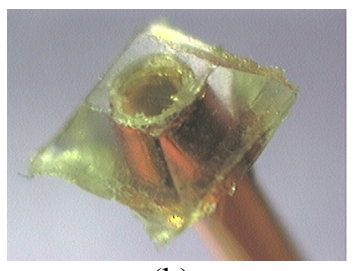

(b)

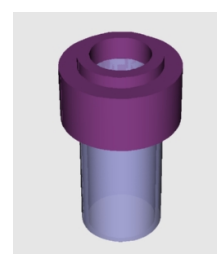

(c)
Figure 2

virtually any cross sectional port or tubing geometry can be matched. Each of these designs incorporates alignment structures, have low dead volume, and only a minimal amount of adhesive material is wetted by fluid flowing through.

\section{Fabrication}

Three types of couplers using three sizes of capillary tubing have been fabricated. Process flows for the various types of couplers are shown in Figures 3-5.

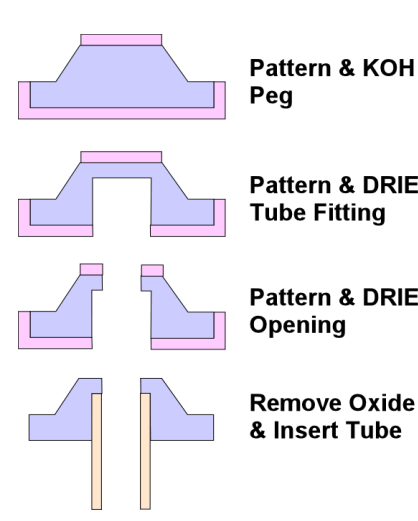

Figure 3

Bulk Coupler Process Flow
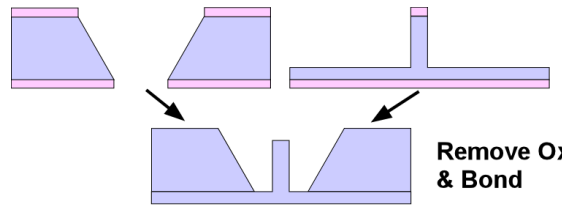

Remove Oxide \& Bond

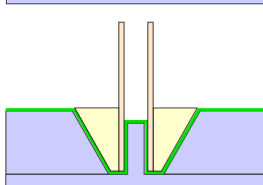

Apply Mold Release, Insert Tube, \& Mold

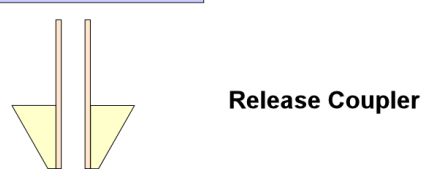

Figure 4

Molded Coupler Process Flow
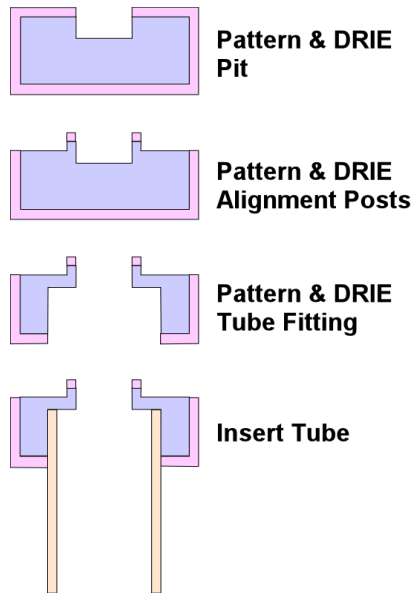

Insert Tube

Figure 5

Post Coupler Process Flow

\section{Bulk Coupler}

Oxidized silicon wafers $(\sim 1.5 \mu \mathrm{m})$ are patterned and etched in $\mathrm{KOH}$ (potassium hydroxide) until pegs of about $270 \mu \mathrm{m}$ high are formed. Press-fit pits for tubing are then etched by DRIE. Depending on the application, a stepped pit can be formed by leaving about $50 \mu \mathrm{m}$ of silicon then etching a smaller diameter hole through the remaining silicon. This additional shoulder forms a tubing stop and is shown in Figure 6.

As evident from the desired geometry, corner compensation is required to fabricate bulk couplers (Figure 2a). Two schemes were investigated and etching results are shown in Figure 7. The merits of each method are described in [6] and [7], respectively. Additional features in Figure $7 \mathrm{~b}$ are artifacts attributed to the DRIE of masking gaps due to poor photoresist step coverage. While both convex corners exhibit a certain degree of undercut, slight modifications to the etch mask and careful attention to etching progress should eliminate these features. 


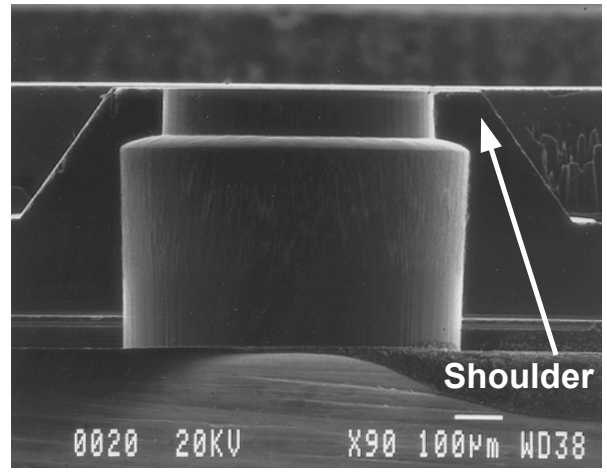

Figure 6

Cross Sectional SEM of Bulk Coupler

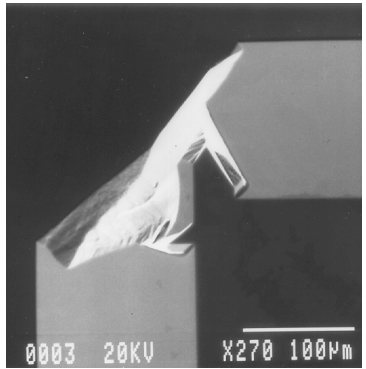

(a)

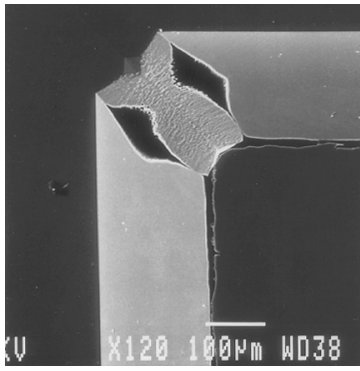

(b)

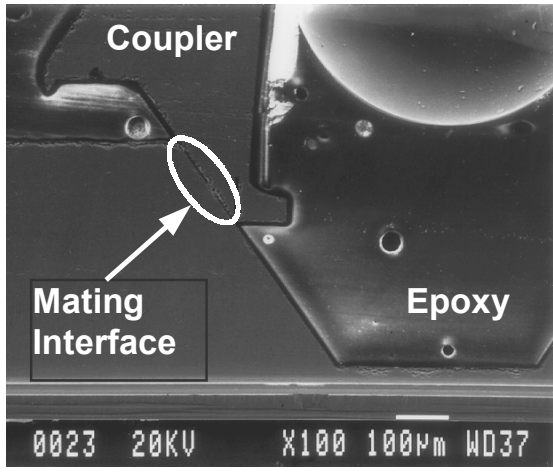

Figure 7

SEM of Bulk Coupler Glued to Plugged Fluidic Port

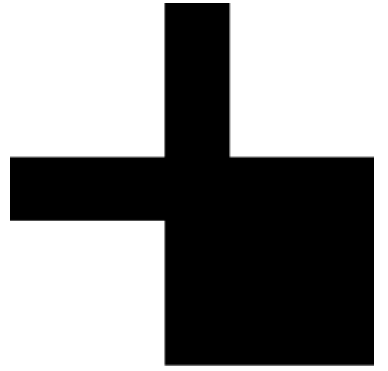

(c)

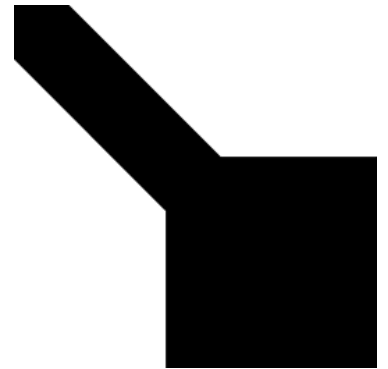

(d)

Figure 8

SEM Corner Compensation Results (a) \& (b) with Respective Masks (c) \& (d)

\section{Molded Bulk Coupler}

Molded bulk couplers (Figure 2b) are fabricated from two bonded wafers. One is an oxidized wafer etched with $\mathrm{KOH}$ through holes and the other wafer is DRIE'd to form an array of circular pegs. After bonding the two wafers together, the structure is coated with a PTFE (polytetrafluoroethylene) based mold release. Moldings are realized by melting raw material such as a hot-melt polyolefin around fused silica tubing fitted on mold posts and releasing when cool. Released structures are then attached to fluidic ports simply by reheating polyolefin, allowing it to reflow and adhere to the silicon port. In applications where heating is unacceptable, adhesive joining can be used.

\section{Post Coupler}

DRIE processing allows flexibility in creating two dimensional geometries across a wafer surface. Thus, post couplers (Figure 2c) can used with virtually any fluidic port and tubing. Here, a generic process flow is described and one for a cylindrical coupler is depicted.

First, an opening is first defined in an oxidized wafer to form a tube stopper. Then alignment structures and tube receptacles are etched. Tubes are press-fitted into the receptacles and joined to a fluidic port.

\section{Testing}

Testing under high pressure conditions was performed using the setup in Figure 9. Valco stainless steel high pressure liquid chromatography (HPLC) fittings and Vespel/graphite ferrules were used to connect a fused silica capillary to conventional Swagelok plumbing. In order to determine the failure point and the operating range, a blocked coupler was connected to fused silica capillary and pressurized using filtered $(0.1 \mu \mathrm{m})$ nitrogen gas from a cylinder. 
Pressure was measured using an Omega PX120-2KGV pressure transducer in conjunction with an Omega DP25-S Strain Gage Panel Meter. The bulk coupler (with $680 \mu \mathrm{m}$ OD fused silica tubing) was able to withstand at least 1200 psi.

The following table provides a comparison of our work to other couplers.

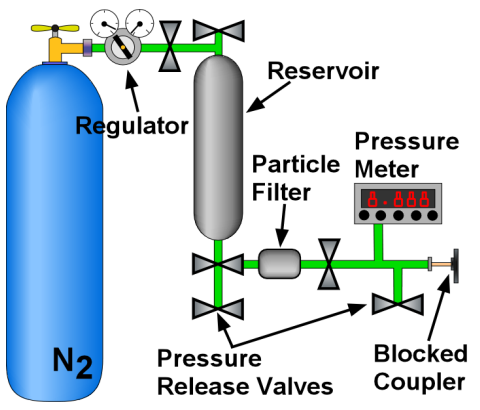

Figure 9

Schematic of Testing Apparatus

\begin{tabular}{|l|c|c|}
\hline \multicolumn{1}{|c|}{ Description of Coupler } & $\begin{array}{c}\text { Operation } \\
\text { Range }\end{array}$ & References \\
\hline $\begin{array}{l}\text { Silicon Finger Microjoint with } \\
\text { Silicone Gasket and Tygon Tubing }\end{array}$ & $>30 \mathrm{psi}$ & {$[8]$} \\
\hline $\begin{array}{l}\text { Silicon/Plastic Coupler with } \\
\text { Silicone Gasket \& Capillary Tubing }\end{array}$ & $\sim 60 \mathrm{psi}$ & {$[2]$} \\
\hline $\begin{array}{l}\text { Silicone Gasket Sealed Silicon } \\
\text { Coupler with Capillary Tubing }\end{array}$ & $\sim 80 \mathrm{psi}$ & {$[4]$} \\
\hline $\begin{array}{l}\text { Silicon Sleeve Coupler with } \\
\text { Capillary Tubing }\end{array}$ & $\sim 500 \mathrm{psi}$ & {$[2]$} \\
\hline $\begin{array}{l}\text { Silicon Coupler with Fused } \\
\text { Capillary Tubing }\end{array}$ & $>1200 \mathrm{psi}$ & This work \\
\hline $\begin{array}{l}\text { Mismatched Silicon Coupler with } \\
\text { Capillary Tubing }\end{array}$ & $\sim 1740 \mathrm{psi}$ & {$[3]$} \\
\hline
\end{tabular}

Table 1

\section{Conclusions}

Micromachined fluidic couplers capable of withstanding up to 1200 psi have been designed, fabricated, and tested. These couplers are robust, have high yield, and are compatible with existing MEMS fluidic devices.

\section{Acknowledgements}

The authors would like to thank Mr. Tuan Hoang for help with proofreading and Mr. Trevor Roper and Mr. Hung Bui for assistance with fabrication.

\section{References}

1. Gonzalez, C., S.D. Collins, and R.L. Smith, Fluidic interconnects for modular assembly of chemical microsystems. Sensors and Actuators B, 1998. 49: p. 40-45.

2. Gray, B.L., et al., Novel interconnection technologies for integrated microfluidic systems. Sensors \& Actuators A Physical, 1999(1): p. 57-65.

3. Spiering, V.L., et al., Novel microstructures and technologies applied in chemical analysis techniques, in Transducers '97. 1997: Chicago, IL. p. 511-514.

4. Yao, T.J., et al., A Micromachined Rubber O-Ring Microfluidic Coupler, in MEMS 2000. 2000: Miyazaki, Japan. p. 624-627.

5. Yang, X., C. Grosjean, and Y.-C. Tai, A Low Power MEMS Silicone/Parylene Valve, in Hilton Head '98. 1998: Hilton Head Island, South Carolina.

6. Jiang, F., et al. A Micromachined Polysilicon Hot-Wire Anemometer. in Solid-State Sensor and Actuator Workshop. 1994. Hilton Head, SC.

7. Enoksson, P., New structure for corner compensation in anisotropic KOH etching. Journal of Micromechanics and Microengineering, 1997. 7(3): p. 141-147.

8. Gonzalez, C., et al., Microjoinery: Concept, Definition, and Application to Microsystem Development. Sensors and Actuators A, 1998. 66(1-3): p. 315-332. 